\title{
IM/DD dual stream asymmetrically clipped optical OFDM system
}

\begin{abstract}
A dual stream asymmetrically clipped optical (DSACO)-orthogonal frequency division multiplexing (OFDM) with intensity modulation/direct detection receiver is proposed that combines the ACO-OFDM, which is generated from individual streams of odd and even frequencies in a single frame without loss of information. The clipping noise, which is its absolute value, is subtracted from even subcarriers of DSACO-OFDM signal and further delayed in time domain to recover the ACO-OFDM data of the odd stream. The proposed DSACO has the same spectral efficiency of DC-biased optical OFDM (DCO-OFDM). It performs better in terms of optical signal-to-noise ratio (OSNR) and peak-to-average power ratio (PAPR) when compared to ASCO-OFDM and Lowery's layered/enhanced ACOOFDM (L/E ACO-OFDM). For a bit error rate of 10 - 3, DSACO shows around 1.9, 3.4, and 7.6 dB improvements of OSNR over two-layered L/E ACO-OFDM, three-layered L/E ACO-OFDM, and ASCO-OFDM for 1024-quadrature amplitude modulation constellations, respectively. The complimentary cumulative distributive function of PAPR for DSACO achieves $0.27 \mathrm{~dB}$ lower PAPR than three-layered L/E ACO-OFDM system and $1.17 \mathrm{~dB}$ lower PAPR than two-layered L/E ACO-OFDM and ASCO-OFDM.
\end{abstract}

Keyword: Orthogonal frequency division multiplexing; Receivers; Signal to noise ratio; Modulation; Interference (communication); Transmitters; Monte Carlo methods . 